\title{
Design of Tractor Resistance Sensor based on Magnetostriction Effect
}

\author{
Mingsheng Li a, Jingyi Gong ${ }^{b}$, Jin Ye ${ }^{c, *}$ and Jian Liu ${ }^{d}$ \\ College of Engineering and Technology, Southwest University, Chongqing 400700, China
}

ali-ming.2004@163.com, b495916189@qq.com, cyejin2007@gmail.com, dswordsheer@qq.com

Corresponding author: Jin Ye

Keywords: High power tractor, Resistance sensor, Magnetostriction, Design, Simulation.

\begin{abstract}
This paper designed the Pin-axis Resistance sensor based on the principle of Magnetostriction. The mathematic model of the sensor was established and simulated in Matlab / Simulink. In this paper , load experiment results of resistance sensor showed that resistance sensor's linearity decision coefficient of the static characteristic curve was 0.9965 . The output voltage was $0.55 \mathrm{~V}$ when the shearing force was $70 \mathrm{kN}$.
\end{abstract}

\section{Introduction}

Raiser is a special component on tractor which is used for raising farm implements and controlling depth of cultivating. Its control performance directly affects the tractor operation quality as well as the operation efficiency [1]. At present, domestic and foreign high-power tractors gradually use electro-hydraulic raiser to realize automatic control in order to improve the control precision and reduce the driver's labor intensity [2 3]. Resistance sensor as a significant component of electro-hydraulic riser plays an important role in its performance.

At present, resistance sensors of high-power tractor electro-hydraulic raiser control system mainly include piezoelectric and resistance strain gauges. Compared with the common resistance strain gauges, magnetostriction sensor has many advantages, such as high output power, strong anti-interference ability, and adapting harsh working environment and suitable for static and dynamic force measurement. Magnetostriction sensor in the field of industrial automation control system has a good prospect [4].

In this paper, the basic principles and characteristics of magnetostriction were expatiated, then the magnetostriction sensor was designed. Finally, the performance simulation and experimental verification were carried out.

\section{Design of Magnetostriction Resistance Sensor}

\subsection{Magnetostriction Effect and Reversible Magnetostriction Effect}

Magnetostriction effect is the magnetostriction material in the magnetic field with the ability to stretch. In addition, the magnetostriction material also has another important effect characteristics -reversible magnetostriction effect. It will cause the permeability of the material changes and thus Change the magnetic field, when the magnetized ferromagnetic body is deformed or subjected to stress [5]. The use of these two effects of magnetic materials can be produced to detect magnetic field, strain, displacement, torque, and pressure, current and other components. Magnetostrictive resistance sensor is the essence of the role of force, the material within the magnetic domain rotation, the magnetic permeability of the material changes, resulting in a change in the magnetic flux density inside and around the material, thus causing the output voltage of the sensor to change.

\subsection{Design and Strength Checking the Shell of Magnetostriction Resistance Sensor}

When the tractor is working, The farm implements by the soil resistance through the lower link act on the resistance sensor.The resistance sensor is subjected to the maximum shear force in the separation surface area of the lower link and the supporting seat and outputs the voltage signal related to the shear force. The resistance sensor's installation form in High-power tractor is shown in Figure 1.Therefore, the design of magnetostriction resistance sensor shell structure is the Pin-axis type. Its force-bearing area 
is a whole hollow cross-section round shaft, which structure is compact, simple geometric shape , easy to process the precise dimensional accuracy and form and position accuracy. Hollow section has a strong ability to resist torsion and bending. The stress of the pin center is the biggest, and it is easy to assemble with relevant bearing parts, easy to use.

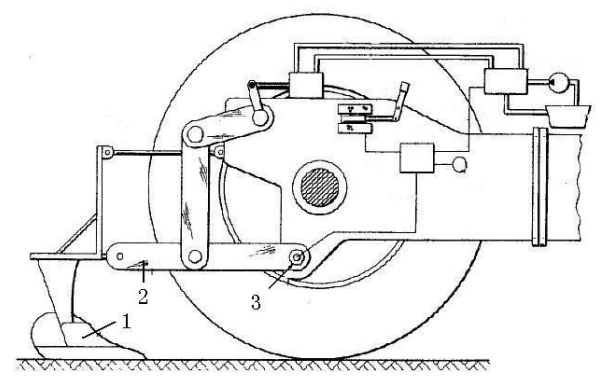

1. Plough 2. Lower link 3. Resistance sensor

Fig. 1 mounting position of force transducer in electronic-controlled hitch system

The maximum shear force that the resistance sensor supporting position can withstand $60 \mathrm{kN}$, so the sensor directly under the force of the pin shell must have sufficient strength. In this paper, two kinds of pins commonly used materials 45 and 40Cr hardened and tempered steel were analyzed for strength. 45 and 40Cr hardened and tempered steel basic parameters as shown in Table 1.

Tab.1 Parameters of 45 and $40 \mathrm{Cr}$ hardened and tempered steel

\begin{tabular}{|c|c|c|c|c|c|}
\hline Material & $\begin{array}{c}\text { Permissible } \\
\text { tensile stress } \\
|\sigma|(\mathrm{MPa})\end{array}$ & $\begin{array}{c}\text { Permissible } \\
\text { bending stress } \\
\left|\sigma_{\max }\right|(\mathrm{MPa})\end{array}$ & $\begin{array}{c}\text { Permissible } \\
\text { shear stress } \\
|\tau|(\mathrm{MPa})\end{array}$ & $\begin{array}{c}\text { Elastic } \\
\text { modulus } \\
\mathrm{E}(\mathrm{GPa})\end{array}$ & $\begin{array}{c}\text { Yield strength } \\
\sigma_{S}(\mathrm{MPa})\end{array}$ \\
\hline Parameters of 45 & 180 & 100 & 126 & $2.1 \times 104$ & 360 \\
\hline $\begin{array}{c}\text { 40Cr hardened } \\
\text { and tempered steel }\end{array}$ & 275 & 120 & 192 & $2.1 \times 104$ & 550 \\
\hline
\end{tabular}

The pin shearing force sensor can be simplified as a hollow section beam with a concentrated loads acting on both ends of the simply supported. Hollow section as shown in Figure 2.

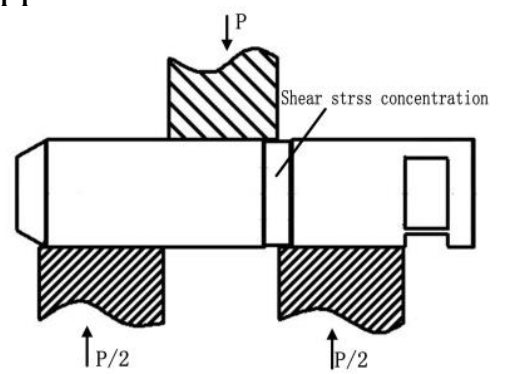

Fig. 2 Force diagram of draft sensor

The shear stress concentration region is the hollow section of the center of the groove. The shear force at the neutral layer is maximum. It can be expressed as follows:

$\tau=\frac{8 P}{3 \pi} \cdot \frac{D^{2}+D d+d^{2}}{D^{4}-d^{4}} \approx 53.56 \mathrm{MPa}$

Pin central layer and axis of the $45^{\circ}$ direction of the principal stress $\sigma$, the principal strain $\varepsilon$ is

$\sigma= \pm \tau= \pm 53.56 \mathrm{MPa}$

$$
\varepsilon= \pm \frac{\gamma \tau}{2 G}= \pm 3316 \times 10^{-7}
$$

The maximum bending stress of the hollow section central layer is

$\sigma_{\max }=\frac{8 P L}{\pi D^{3}\left[1-\left(\frac{d}{D}\right)^{4}\right]} \approx 34.93 \mathrm{MPa}$

To sum up, $[\sigma]<[\sigma] 45<[\sigma] 40 \mathrm{Cr} ;[\tau]<[\tau] 45<[\tau] 40 \mathrm{Cr}$; $[\sigma \max ]<[\sigma \max ] 45<[\sigma \max ] 40 \mathrm{Cr}$

Therefore, both materials meet the strength requirements. 


\subsection{Design of Internal Magnetic Circuit of Magnetostrictive Resistance Transducer}

As the magnetic flux changes in the magnetic circuit affect the output signal of the sensor, so the magnetic circuit directly affects the size of the sensor output signal. The design of magnetic circuit should minimize magnetic leakage and improve electromagnetic transform efficiency. Try to avoid parasitic magnetic field generated during components operation, Such as eddy magnetic field by eddy current generated, and improving the uniformity of the magnetic field in which the magnetic material is located. The maximum wall thickness of the pin resistance sensor is less than $10 \mathrm{~mm}$, so the coil exciting current using DC pulsating current, by changing the size of the input current can change the size of the magnetic field. The design of the excitation circuit is shown in Figure 3. When using DC excitation, the use of lamellar, the appropriate gap between the two layers can reduce the inductance of the coil, to avoid DC saturation of the core, to avoid core saturation due to direct current. The design of sensor magnetic circuit is shown in Figure 4.

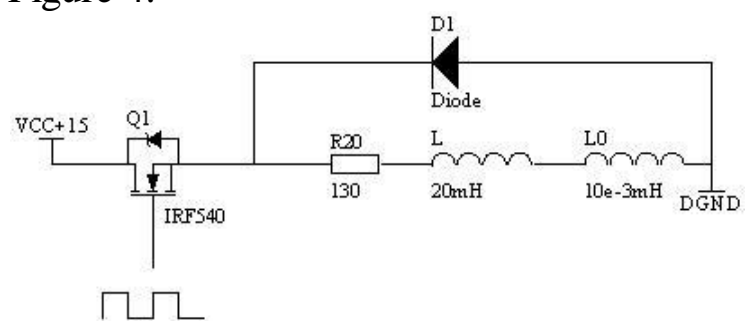

Fig.3 Schematic diagram of excitation circuit
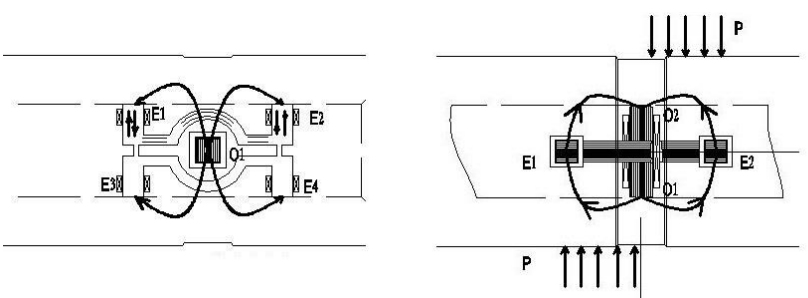

Fig.4 Magnetic circuit of draft sensor

In Figure 4, when the resistance sensor is not force, the primary coil between the two poles form a symmetrical magnetic field. The reluctance of each segment on the sensor surface is equal and the flux through the induction coil is equal. As the coil E1 and E2 reverse series, E3 and E4 reverse series, two groups of coils are connected in series to form the differential connection. So the magnetic flux cancel each other out, the sensor output is zero. When the sensor is subjected to shear forces, the magnetic properties of the natural isotropic magnetic material will change. As a result, the magnetic field becomes asymmetric, producing tensile and compressive stresses in the $45^{\circ}$ and $135^{\circ}$ directions, as shown in Figure 4.

According to ferromagnetism, when the saturation magnetostriction of steel material is greater than zero $\lambda_{S}>0$. The magnetoelastic in the tensile stress direction is the lowest, which is the easy magnetization direction, that the permeability increases and the reluctance decreases. However, in the compressive stress direction, the permeability decreases and the reluctance increases. Therefore, E1, E2, E3, E4 of the magnetic flux change, the sensor has a corresponding induced voltage output.

Magnetic core material can affect the magnetic field of magnetic induction, the choice of materials require high permeability, low loss, wide magnetization curve linear segments and the magnetic properties stability of the materials. Commonly used magnetic core material perm alloy $1 \mathrm{~J} 85$ and silicon steel sheet B30P110 control parameters shown in Table 2. Comparison shows that, compared with silicon steel sheet B30P110, Perm alloy 1J85 has very low coercively, high initial permeability and maximum permeability, sensitive to weak signals. Saturation magnetic induction intensity is low, so the choice of perm alloy $1 \mathrm{~J} 85$ as the core material. 
Tab.2 Parameters of magnetic core materials

\begin{tabular}{|c|c|c|c|c|}
\hline Material(0.03mm) & $\begin{array}{c}\text { The initial relative } \\
\text { permeability(Gs/Oe) }\end{array}$ & $\begin{array}{c}\text { The maximum relative } \\
\text { magnetic permeability(Gs/Oe) }\end{array}$ & $\begin{array}{c}\text { Saturation } \\
\text { magnetostriction } \\
\text { constant }\end{array}$ & $\begin{array}{c}\text { Coercivity } \\
\mathrm{MPa}\end{array}$ \\
\hline perm alloy 1J85 & $4 \times 104$ & $1.8 \times 105$ & $<2 \times 10-6$ & $\leq 1.2$ \\
\hline $\begin{array}{c}\text { silicon steel sheet } \\
\text { B30P110 }\end{array}$ & $1 \times 103$ & $4 \times 104$ & $1 \times 10-5$ & $>8$ \\
\hline
\end{tabular}

\section{Modeling and Simulation of Magnetostrictive Resistance Sensor}

According to the theory of ferromagnetism, the relationship between the relative permeability of the piezomagnetic material with the stress is

$$
\Delta \mu=2 \frac{\lambda_{s}}{B_{S}^{2}} \mu^{2}\left(\frac{8 P}{3 \pi} \frac{D^{2}+D d+D^{2}}{D^{4}-d^{4}}\right)
$$

According to the working principle of the resistance sensor, the magnetic circuit of the sensor can be equivalent to the equivalent magnetic circuit shown in Fig5. R0 is the resistance of the magnetic flux through the excitation magnetic poles. R1, R2, R3, R4 are the magnetic flux through the four induction magnetic reluctance including induction magnetic core reluctance, Gap Reluctance between induction magnetic pole and measured surface and the corresponding measured surface segment magnetoresistance. R5 is the magnetic resistance of the part of the magnetic flux on the surface of the material which has not passed through the induction magnetic pole. Due to flow through R5 flux $\Phi 5$ is relatively small, it is negligible.

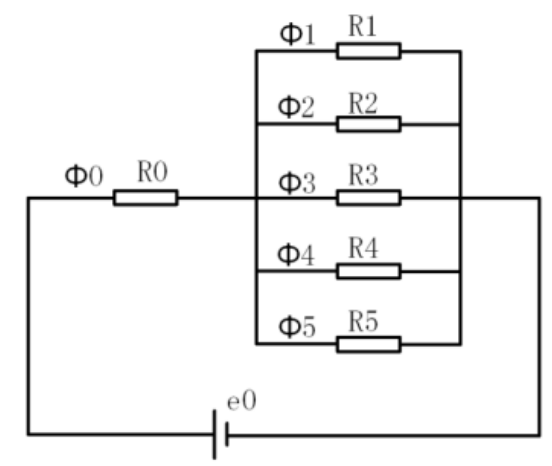

Fig.5 Equivalent magnetic circuit of draft sensor

When without applying force to the center pin or at rest, it is known that the magnetic circuit symmetric $\Phi 1=\Phi 2=\Phi 3=\Phi 4$. According to the principle of flux continuity, $\Phi 0=\Phi 1+\Phi 2+\Phi 3+$ $\Phi 4$.

According to amperes and Magnetic circuit Ohm's Law,

$$
\Phi_{1}=\frac{N_{0} I_{0}}{\frac{4 l_{0}}{\mu_{M} S_{0}}+\frac{l_{1}}{\mu_{M} S_{1}}+\frac{l_{C 1 O}}{\mu_{C} S_{C 1 O}}+\frac{l_{C O 1}}{\mu_{C} S_{C O 1}}+\frac{2 \delta_{0}}{\mu_{0} S_{0}}+\frac{2 \delta_{1}}{\mu_{0} S_{1}}}
$$

According to the magnetic circuit symmetry, $l_{C 1 O} \approx l_{C O 1}, S_{C 1 O} \approx S_{C O 1}, \delta_{0} \approx \delta_{1}$, then

$$
\begin{aligned}
& K_{M}=\frac{1}{A+B+\frac{C}{\mu_{C}}} \\
& \Phi_{1}=K_{M} N_{0} I_{0}
\end{aligned}
$$

Among them $A=\frac{4 l_{0}}{\mu_{M} S_{0}}+\frac{l_{1}}{\mu_{M} S_{1}} B=\frac{145}{32 \mu_{0} S_{0}} \delta_{0} C=\frac{2 l_{C 10}}{S_{C 10}}$

When the sensor is subjected to shear forces, tensile stress is generated in the $45^{\circ}$ and $135^{\circ}$ directions of the surface. The corresponding permeability of the surface respectively increases or decreases $\Delta \mu \mathrm{C}$, then the induction voltage of the four induction coils is $\Delta U$ and $-\Delta U$. According to the induction core 
symmetry, E1 coil flows through $\Phi 1$ and $\Phi 2, \Phi 1$ and $\Phi 2$ in the opposite direction, and the force is shown in Figure 4, then

$$
\begin{aligned}
& \mu_{C 1}=\mu_{C}-\Delta \mu \\
& \mu_{C 2}=\mu_{C}+\Delta \mu
\end{aligned}
$$

Reduce $\Phi 1$, increase $\Phi 2$, then

$e_{1}=-N_{1} \frac{d\left(\Phi_{1}-\Phi_{2}\right)}{d t}=-N_{1} \frac{d \Phi_{1}}{d t}+N_{1} \frac{d \Phi_{2}}{d t}$

As the absolute value of tensile stress is equal, $\frac{d \mu_{C 1}}{d t}=-\frac{d \mu_{C 2}}{d t}$, and the core and the axis is transition fit, The effect of air gap change is relatively small, $\delta_{0}$ can be regarded as constant, then the coil E1 induced electromotive force is

$$
e_{1}=N_{0} N_{1}\left(K_{M 2}-K_{M 1}\right) \frac{d I_{0}}{d t}+N_{1} K \frac{d \mu_{C 1}}{d t}
$$

Similarly, E2, E3, E4 induced electromotive force is

$$
e_{2}=N_{0} N_{2}\left(K_{M 1}-K_{M 2}\right) \frac{d I_{0}}{d t}+N_{2} K \frac{d \mu_{C 1}}{d t} ; \quad e_{3}=N_{0} N_{3}\left(K_{M 2}-K_{M 1}\right) \frac{d I_{0}}{d t}+N_{3} K \frac{d \mu_{C 1}}{d t} ; e_{4}=N_{0} N_{4}\left(K_{M 1}-K_{M 2}\right) \frac{d I_{0}}{d t}+N_{4} K \frac{d \mu_{C 1}}{d t}
$$

Among them, $K=C N_{0} I_{0}\left(\frac{K_{M 1}}{\mu_{C 1}{ }^{2}}+\frac{K_{M 2}}{\mu_{C 2}{ }^{2}}\right) \quad K_{M 1}=\frac{1}{A+B+\frac{C}{\mu_{C 1}}} K_{M 2}=\frac{1}{A+B+\frac{C}{\mu_{C 2}}}$

The inductive coil is a differential connection, so the output voltage of the sensor is

$$
\vec{e}_{\text {OUT }}=\vec{e}_{1}-\vec{e}_{2}+\vec{e}_{3}-\vec{e}_{4}=N_{0}\left(N_{1}+N_{2}+N_{3}+N_{4}\right)\left(K_{M 2}-K_{M 1}\right) \frac{d I_{0}}{d t}+K\left(N_{1}+N_{2}+N_{3}+N_{4}\right) \frac{d \mu_{C 1}}{d t}
$$

When a static force is applied, the output voltage of the sensor is

$$
\vec{e}_{\text {OUT }}=\vec{e}_{1}-\vec{e}_{2}+\vec{e}_{3}-\vec{e}_{4}=N_{0}\left(N_{1}+N_{2}+N_{3}+N_{4}\right)\left(K_{M 2}-K_{M 1}\right) \frac{d I_{0}}{d t}
$$

The excitation circuit is modeled by Matlab, and the resistance sensor is simulated. Simulation results shown in Fig.6, Fig.6 shows that with the increase of shear force, the sensor output voltage is also an upward trend, and tends to a linear variation relationship; when the shear force tends to be stable, the sensor output voltage tends to be stable.

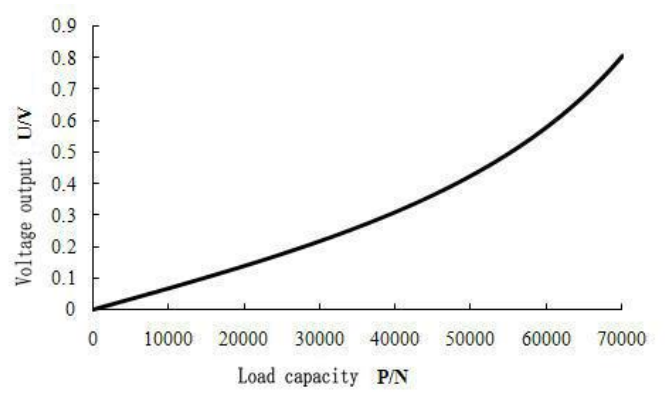

Fig.6 Simulation curve of draft sensor

\section{Performance test of magnetostrictive resistance sensor}

In order to test the static characteristics of the magnetostrictive resistance sensor, Shear force test of 45 and 40Cr hardened and tempered steel shells sensor using material testing machine, As shown in Fig 7. The maximum force of the designed sensor is $60 \mathrm{kN}$, and the maximum loading force of the testing machine is $70 \mathrm{kN}$.

The static characteristic curves of the 45 and $40 \mathrm{Cr}$ hardened and tempered steel shells resistance sensor are shown in Fig7 As can be seen from Fig. 7, the determination coefficient $\mathrm{R}^{2}$ of the 45 hardened and tempered steel modulation resistance sensor is 0.9965 . The output voltage is $0.55 \mathrm{~V}$ when $70 \mathrm{kN}$ shearing force is applied. $40 \mathrm{Cr}$ hardened and tempered steel shell resistance sensor determination 
coefficient $\mathrm{R}^{2}$ is 0.9872 . The output voltage is $0.39 \mathrm{~V}$ when $70 \mathrm{kN}$ shearing force is applied. So 45 hardened and tempered steel performance is better than $40 \mathrm{Cr}$ hardened and tempered steel.

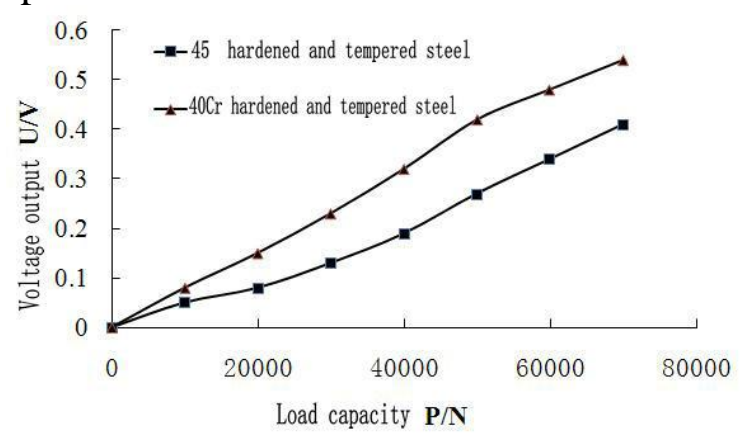

Fig.7 Draft sensor characteristic curve

The voltage output signal of the resistance sensor has a linear relationship with the loading shear force. Nonlinear factors are mainly due to the sensor coil is manually wound, winding tightness, arrangement and coil turning point of its performance may have an impact. When the coil standardization of mechanical manufacturing can reduce the coil instability. At the same time, in the limited space within the pin hole limited, the standardization of mechanical winding coil can be increased, the magnetization also can be increased.

\section{Summary}

(1) Based on the magnetostrictive principle of the pin sensor design, the use of perm alloy $1 \mathrm{~J} 85$ as the core material, shell material selection 45 and $40 \mathrm{Cr}$ hardened and tempered steel. Sensor excitation circuit is DC pulse excitation. The simulation results show that the sensor has good force-voltage conversion characteristics.

(2) The load test results of the sensor showed that 45 hardened and tempered steel shell static characteristic curve linearity determination coefficient $\mathrm{R}^{2}$ is 0.996 , the maximum output voltage is $0.55 \mathrm{~V}$. $40 \mathrm{Cr}$ hardened and tempered steel shell static characteristic curve linearity determination coefficient $\mathrm{R}^{2}$ is 0.9827 , the maximum output voltage is $0.39 \mathrm{~V}$.

\section{Acknowledgements}

This paper is supported by the project: Fundamental Research Funds for the Central Universities (XDJK2016B007) and Research Foundation for Doctor (SWU115064).

\section{References}

[1] Li Mingsheng, Zhu Zhongxiang, Mao Enrong. Design of proportional raise valve in electro-hydraulic lifting mechanism of big-power tractor $[\mathrm{J}]$. Transactions of the Chinese society for agricultural machinery: 2012, 43(10): 31 35.

[2]Weatherly E T, Bowers Jr C G. Automatic depth control of a seed planter based on soil drying front sensing [J]. Transactions of the ASAE, 1997, 40(2): 295 305.

[3]Yin Xiujie, Song Zhenghe, Zhu Zhongxiang, et al. New electro-hydraulic control system for tractor [J]. Journal of Jilin University : Engineering and Technology Edition, 2008, 38(3): 580 585.

[4]Yan Rongge, Yang Qingxin, Yang Wenrong, et al. Giant magnetostrictive acceleration sensors [J]. Proceedings of the CSEE, 2009, 29(24): 104 109.

[5].Li Songtao, Meng Fanbin, Liu Heyan, et al. Giant magnetostrictive materials and their application [J]. Physics, 2004, 33(10): 748 752. 